\title{
HARWELL RADIOCARBON MEASUREMENTS V
}

\author{
A J WALKER, R S KEYZOR*, and R L OTLET
}

Isotope Measurements Laboratory, Nuclear Applications Centre, Atomic Energy Research Establishment, Harwell, Oxfordshire, OX11 ORA, UK

\section{INTRODUCTION}

The results presented in this list include some recently measured samples (1984) but mostly ones from our earlier years of operation which had not been previously published in RADIOCARBON. It is the first of a number of special lists prepared over the last year so that the backlog of unpublished dates of this laboratory will be cleared. The samples are all archaeologic from the United Kingdom most of which have originated from "rescue"-type excavations.

As in previous lists, all samples were measured by liquid scintillation counting (Otlet \& Warchal, 1978), and the error term quoted is the $1 \sigma$ standard deviation estimate of the full replicate sample reproducibility (Otlet, 1979). The list was produced semi-automatically from the Harwell data base stored on the main frame computer using the procedures described in Otlet and Walker (1983). Calculations are based on the Libby half-life of 5568 years, using NBS oxalic acid standard (x0.95) as "modern," both values treated as constants, with AD 1950 as the reference year. All results are corrected for fractionation according to the quoted $\delta^{13} \mathrm{C}$ (wrt PDB) values measured in this laboratory.

\section{ACKNOWLEDGMENTS}

We wish to acknowledge the work of our colleagues, G A Bradburn and D G Humphreys, with the laboratory measurements and of E F Westall, S E Hasler, and M Gibson with the preparation of the data in computer readable form. The financial support and cooperation of the staff of the Historic Buildings and Monuments Commission for England (Ancient Monuments Laboratory), through which most of the samples (those additionally referenced with AML numbers) were submitted, is also gratefully acknowledged.

\section{ARCHAEOLOGIC SAMPLES}

British Isles

\section{England}

\section{Stonehenge Car Park series}

Charcoal samples, presumably decayed bark, from Stonehenge Car Park, Stonehenge, Salisbury, Wiltshire $\left(51^{\circ} 10^{\prime} 49^{\prime \prime} \mathrm{N}, 1^{\circ} 49^{\prime} 37^{\prime \prime} \mathrm{W}\right.$, Natl Grid Ref SU 121424). Coll Feb 1966 by H L and F J de M Vatcher and subm by H Keeley, Ancient Monuments Lab. For description of excavation see Anonymous (1973).

\footnotetext{
* also School of Archaeologic Sciences, University of Bradford, UK
} 
HAR-455. HK1

$9130 \pm 180$

$\delta^{13} C=-24.2 \%$

From Hole A, depth $0.76 \mathrm{~m}$, halfway between top (natural chalk) and base, on post circumference.

HAR-456. HK4

$\mathbf{8 0 9 0} \pm \mathbf{1 4 0}$
$\delta^{13} C=-25.4 \%$

AML 733250, from Hole B, depth $0.91 \mathrm{~m}$ from surface of natural chalk.

General Comment (FJdeMV): dates are unexpectedly early; we do not know why this post hole is ca $1000 \mathrm{yr}$ younger than the other which should have been contemporaneous. Excavator thinks that samples, although pertaining to original posts, were of poor quality, being fine and mixed with other material.

\section{Marc 3 series}

Samples from two sites described in Fasham (1980), R6, Burntwood Farm and R5, Bridget's Farm, both in Itchen Valley Parish, Hampshire along planned route of M 3 Motorway. Previous dates from Rte M 3 are reported in Walker and Otlet (1985, p 75-76). All samples coll and subm by P J Fasham, Archaeol Rescue Comm, Winchester.

HAR-1021. R6-801

$$
\begin{array}{r}
1980 \pm 110 \\
\delta^{13} C=-19.6 \% 0
\end{array}
$$

Human leg bones, AML 749329, from grave 55 at Burntwood Farm $\left(51^{\circ} 6^{\prime} 13^{\prime \prime} \mathrm{N}, 1^{\circ} 16^{\prime} 11^{\prime \prime} \mathrm{W}\right.$, Natl Grid Ref SU 51133411). Coll Oct 1974 and subm Nov 1974. Comment: uncertainties quoted may underestimate actual measurement errors due to low laboratory yields.

HAR-1695. R5-816

$$
\begin{array}{r}
\mathbf{3 7 9 0} \pm \mathbf{7 0} \\
\delta^{13} C=-24.5 \%
\end{array}
$$

Charcoal, nut shells, AML 750725, from Bridget's Farm $\left(51^{\circ} 6^{\prime} 26^{\prime \prime} \mathrm{N}\right.$, $1^{\circ} 15^{\prime} 58^{\prime \prime} \mathrm{W}$, Natl Grid Ref SU 51373453). Coll Dec 1974 and subm May 1976 .

HAR-2745. R5-817

$$
\begin{array}{r}
\mathbf{2 8 0 0} \pm \mathbf{8 0} \\
\delta^{13} C=-24.2 \% 0
\end{array}
$$

Bone, AML 780963, from Bridget's farm $\left(51^{\circ} 6^{\prime} 26^{\prime \prime} \mathrm{N}, 1^{\circ} 15^{\prime} 58^{\prime \prime} \mathrm{W}\right.$, Natl Grid Ref SU 51373453). Coll Dec 1974 and subm April 1978.

General Comment (PJF): HAR-1021 formed part of study of close dating system for Iron Age and Roman pottery in area; HAR-1695 and -2745 were intended to date series of otherwise undated pits at Bridget's Farm.

\section{Burton Fleming series}

Human bone, from Iron Age cemetery at Burton Fleming, Yorkshire (54 $4^{\circ} 41^{\prime \prime} \mathrm{N}, 0^{\circ} 19^{\prime} 24^{\prime \prime} \mathrm{W}$, Natl Grid Ref TA 096697). Coll July 1974 and 
subm Jan 1975 by I M Stead, Brit Mus. For description of site see Stead (1977).

\section{HAR-1057. FAAM4}

AML 748109, 1 femur/1 fibula.

HAR-1058. FAAQ10

AML 748110, 1 femur/1 fibula.

\section{HAR-1129. FACM34}

AML 748112.

\section{HAR-1130. FABG7}

AML 748111, 1 femur/1 humerus. Comment: small sample size accounts for large error.

\section{Bishophill series}

Samples from Bishophill I, 58 and 59 Skeldergate, York, North Yorkshire $\left(53^{\circ} 57^{\prime} 20^{\prime \prime} \mathrm{N}, 1^{\circ} 4^{\prime} 56^{\prime \prime} \mathrm{W}\right.$, Natl Grid Ref SE 60215145). Coll by S Donaghey and subm by $\mathrm{H}$ K Kenward, Environmental Archaeol Unit, Univ York. Comments supplied by P V Addyman and R A Hall. Site was excavated by York Archaeol Trust 1973-1975 and yielded evidence of virtually uninterrupted occupation sequence from Roman period to present day. For details of Anglo-Scandinavian phase see Donaghey (1986); for Roman structurès dated here see Carver, Donaghey and Sumpter (1978); for biological remains assoc with Roman structures see Hall, Kenward and Williams (1980).

\section{HAR-1412. BH155}

$$
\begin{array}{r}
1230 \pm 80 \\
\delta^{13} C=-26.0 \%
\end{array}
$$

Charcoal, AML 757497, id by C A Keepax as oak (Quercus sp) and willow (Salix sp) of fairly large timbers from occupation deposit, context 2131. Subm Dec 1975. Comment (RAH): date is compatible with mid-8th century coin from deposit, but pottery in assoc with structure D was 10 th century as was beam in slot marking one wall of structure, HAR-1 728 (Walker \& Otlet, 1985, p 83). Radiocarbon date does not necessarily contradict the later date as it reflects age of timber.

\section{HAR-1416. BH198}

$$
\begin{array}{r}
1840 \pm 70 \\
\delta^{13} C=-27.2 \%
\end{array}
$$

Wood, AML 757498, id by C. A Keepax as oak (Quercus sp), from top part of buried soil, context 2356. Subm Dec 1975. 


\section{HAR-1417. BH201}

$$
\begin{array}{r}
1820 \pm 70 \\
\delta^{13} C=-26.7 \%
\end{array}
$$

Charcoal, AML 757499, id by C A Keepax as oak (Quercus sp) from fragments of fairly large timbers, from rich layer overlying buried soil, context 2360. Subm Dec 1975.

General Comment (PVA): HAR-1416 and -1417 confirm clearance of area in early Roman period preceding primary occupation and now allow reconstruction of local ecological conditions from assoc biological remains and soil evidence.

\section{HAR-1418. BH202}

$$
1930 \pm 70
$$

Charcoal, AML 757500, id by C A Keepax as possibly hazel, from fairly large timbers, from layer assoc with Roman destruction levels, context 2361. Subm Dec 1975. Comment (PVA): early date is surprising as deposit overlay 3rd century Roman levels. Charcoal was presumably derived from trees of considerable age.

\section{HAR-1729. BH8068}

$$
1750 \pm 80
$$

Wood, AML 760237, from timber post in construction pit of Roman well. Subm Feb 1976. Comment (PVA): result accords with estimated date of construction for the Roman well in 2nd to 3rd century AD.

\section{HAR-1866. BH220}

$1840 \pm 60$ 1976.

Charcoal, AML 766965, from lowest fills of Roman well. Subm Nov

HAR-1927. BH300

$$
\begin{array}{r}
1830 \pm 70 \\
\delta^{13} C=-26.4 \% 0
\end{array}
$$

Oak, AML 766963, from timber lining of Roman well. Subm Nov 1976. Comment (HKK): ca 50 rings in sample.

\section{HAR-1928. BH301}

$$
\begin{array}{r}
\mathbf{2 1 5 0} \pm \mathbf{7 0} \\
\delta^{13} C=-27.7 \% 0
\end{array}
$$

Oak, AML 766964, from timber lining of Roman well. Subm Nov 1976. Comments (HKK): ca 50 widely-spaced rings in sample; (PVA): early date suggests re-use of old timbers.

\section{Winklebury series} $83)$.

Continuation of series first reported in Walker and Otlet (1985, p

HAR-1765. 13646

$$
\begin{array}{r}
1930 \pm 70 \\
\delta^{13} C=-25.0 \%
\end{array}
$$

Charcoal, AML 766704, from next to bottom layer in pit at Winklebury Camp, Basingstoke, Hants $\left(51^{\circ} 16^{\prime} 17^{\prime \prime} \mathrm{N}, 1^{\circ} 7^{\prime} 14^{\prime \prime} \mathrm{W}\right.$, Natl Grid Ref 
SU 61355290). Coll by D Bartlett and subm Sept 1976 by G Wainwright. Comment (GW): date does not agree (Smith, 1979) with implication of pot of 6 th to 5 th centuries BC found in same pit.

\section{Poundbury series}

Charcoal from post-Roman settlement at Poundbury, Dorchester, Dorset $\left(50^{\circ} 41^{\prime} 5^{\prime \prime} \mathrm{N}, 2^{\circ} 26^{\prime} 47^{\prime \prime} \mathrm{W}\right.$, Natl Grid Ref SY 685911). All samples coll and subm by C J Sparey Green, Dorchester Excavation Comm, Dorset. Continuation of series first reported (Otlet \& Walker, 1979, p 365); for discussion see Green $(1971,1974,1976,1982)$.

HAR-2281. PC76E276

$1590 \pm 80$

AML 767534, from deposit of dark brown soil and chalk flecks in base of pit, E276. Coll Aug 1976 and subm Sept 1976. Comment (CJSG): date is slightly earlier than expected since sample derived from settlement overlying Roman cemetery still in use in late 4th century AD. Archaeologically settlement might be 5 th to 8 th century $\mathrm{AD}$ but close relationship of structures to cemetery could support original occupation soon after cemetery went out of use. Wood from which this charcoal was derived could have been of some age at time of burial.

\section{HAR-3080. OB12}

$1490 \pm 80$

Additionally referenced as PC71B, J9, 18, 115, AML 790638, from burned layer in base of corn drying oven B 115.

\section{HAR-3081. OB21}

$1530 \pm 60$

$\delta^{13} C=-24.3 \%$

Additionally referenced PC72E, 53, AML 790640, charred grain, from burned layer in base of corn drying oven E 53 .

General Comment (CJSG): these 3 samples provide date for early postRoman settlement on site of Late Roman cemetery which, from coin evidence, continued in use to at least AD 380. They imply rapid change in land use at end of Roman period and date for occupation well before arrival of Saxons (ca AD 650) in this area. Saxon settlements have been identified on several sites in S Britain over last decade, but identification of specifically sub-Roman settlement fills major gap in settlement history of S Britain.

\section{Aldwark series}

Wood, from brushwood at base of fortress rampart at site adjacent to 1 to 5 Aldwark, York, North Yorkshire ( $53^{\circ} 57^{\prime} 43^{\prime \prime} \mathrm{N}, 1^{\circ} 4^{\prime} 36^{\prime \prime} \mathrm{W}$, Natl Grid Ref SE 60585219$)$. Coll by H MacGregor and subm June 1977 by H K Kenward, Environmental Archaeol Unit, Univ York. Comments by R A Hall.

HAR-2300. ALDW/52

AML 776296. 
HAR-2301. ALDW49A

AML 770035.

\section{HAR-2302. ALDW49}

AML 770034

General Comment (RAH): on artifactual evidence rampart is probably 11 th century $\mathrm{AD}$ which agrees well with ${ }^{14} \mathrm{C}$ dates. Rampart is ca $10 \mathrm{~m} \mathrm{SW}$ of Roman NE fortress wall and has either cut away Roman deposits or has been added to Roman rampart as part of new phase of defense work. Bank itself has been cut away by large feature of unknown function and date which was backfilled in 13th century (MacGregor, in press).

\section{Rivenhall series}

Samples from churchyard and church at Rivenhall, Essex $\left(51^{\circ} 49^{\prime}\right.$ $42^{\prime \prime}$ N, 0 39' 11" E, Natl Grid Ref TL 828178). Coll Aug 1972 (HAR-2427) and Aug 1973 and subm Aug 1977 by W J Rodwell, Old Vicarage, Chilcompton, Somerset unless otherwise stated. For description of site see Rodwell and Rodwell (1985).

\section{HAR-2326. GR298}

$$
820 \pm 60
$$

Bone, AML 766978, from cemetery area 1 in churchyard. Subm Nov 1976 by P J Drury. Comment (WJR): one of series of graves forming part of regularly laid out Saxon cemetery. Stratigraphically earlier than Saxon or Medieval cemetery bldg of unknown function, but later than Grave 326 (HAR-2404) below.

HAR-2404. GR326

$$
\begin{array}{r}
1140 \pm 70 \\
\delta^{13} C=-19.6 \%
\end{array}
$$

Bone, AML 766980, from cemetery area 1 in churchyard. Subm Nov 1976 by P J Drury. Comment (WJR): one of deep and early, possibly Middle Saxon, graves in area. Part of regularly planned cemetery.

HAR-2427. $\quad$ R9

$$
\begin{array}{r}
\mathbf{9 5 0} \pm \mathbf{6 0} \\
\delta^{13} C=-26.2 \% 0
\end{array}
$$

Wood, AML 776990, id by C A Keepax as oak, from Medieval church. Comment (WJR): sample was sealed in 14th century blocking of late Saxon window and is thought to be part of original window sill.

\section{Hambledon Hill series}

Charcoal from 1976 excavation season at Neolithic causewayed enclosure, Hambledon Hill, Dorset $\left(50^{\circ} 51^{\prime} 34^{\prime \prime} \mathrm{N}, 2^{\circ} 12^{\prime} 38^{\prime \prime} \mathrm{W}\right.$, Natl Grid Ref ST 852123). All coll Sept/Oct 1976 and subm Sept 1977 by R Mercer, Dept Archaeol, Univ Edinburgh. For other samples in series see Walker 
and Otlet (1985, p 85-86). For description of site see Mercer (1980, 1985).

HAR-2371. HH7647

$$
\begin{array}{r}
4680 \pm 110 \\
\delta^{13} C=-27.5 \%
\end{array}
$$

AML 777006, Site L, Outwork Ditch 1, Layer 7. Comments: small sample size accounts for large error. (RM): burned area seemed to have definite $\mathrm{N}$ edge abutting Layer 9 . Deposit contained bone, flint, and human skeletal material; charcoal fragments were contained within other burned matter.

\section{HAR-2377. HH7679}

$4610 \pm 90$ bers, from Site G, ditch segment 3, Layer $9 \mathrm{~A}$.

\section{HAR-2378. HH7662}

$$
\delta^{13} C=-25.4 \%
$$

AML 777010, 50\% of sample id by C A Keepax as oak and hazel/alder, Site K, outwork ditch 1, Layer 7. Comments: small sample size accounts for large error; (RM): sample came from very ashy area containing charcoal lumps.

General Comment (RM): samples represent 2 layers, 7 (HAR-2371, HAR2378) and 9 (HAR-2377). Layer 7 overlay primary silt layers 8 and 9 and was composed of vacuous chalk lumps and flint nodules. Layer 9 was characterized by gray charcoal silt in deep bowl-shaped pit.

\section{Billingborough series}

Charcoal, from Bronze Age settlement, Billingborough, Lincolnshire $\left(52^{\circ} 53^{\prime} 9^{\prime \prime} \mathrm{N}, 0^{\circ} 19^{\prime} 35^{\prime \prime} \mathrm{W}\right.$, Natl Grid Ref TF 126334). Coll Oct 1977 and subm Dec 1977 by P Chowne, South Lincolnshire Archaeol Unit. For description of site see Chowne (1980).

HAR-2483. BFE77F98 $2390 \pm 70$

$$
\delta^{13} C=-24.8 \% \text { o }
$$
post.

AML 777901, id by C A Keepax as oak (large timber), from charred

\section{HAR-2523. BFE77F43}

$$
\begin{array}{r}
\mathbf{2 4 1 0} \pm \mathbf{8 0} \\
\delta^{13} C=-26.1 \% 0
\end{array}
$$

AML 78009, id by C A Keepax as mainly hazel/alder with some hawthorn (large timbers), from upper levels of silted-up enclosure ditch.

\section{Custom House series}

Waterlogged wood samples, id as oak, part of 315-yr tree-ring sequence, from beams forming timber quay of Roman waterfront at Custom House, London $\left(51^{\circ} 30^{\prime} 26^{\prime \prime} \mathrm{N}, 0^{\circ} 4^{\prime} 45^{\prime \prime} \mathrm{W}\right.$, Natl Grid Ref TQ 33298050). Coll 1973 by Dept Urban Archaeol, London and subm Feb 
1978 by J Hillam, Dept Prehist \& Archaeol, Univ Sheffield. For description of site see Tatton-Brown (1974).

HAR-2532. CUS 1

$1970 \pm 70$

AML 780045. Comment $(\mathrm{JH})$ : years 91-110, growth allowance 220 years.

HAR-2530. CUS 2

$1820 \pm 70$

AML 780046. Comment (JH): years 124-133, growth allowance 190 years.

HAR-2534. CUS 3

$1900 \pm 70$

AML 780047. Comment (JH): years 167-186, growth allowance 145 years.

General Comment $(\mathrm{JH})$ : with growth allowances shown samples can be correlated with further Roman waterfront structures at New Fresh Wharf/Seal House (assoc dates HAR-1 864, -1 865, -1867, -1868; Walker \& Otlet, 1985, p 84). From tree rings Custom House structure appears a few decades earlier than this; for full dendrochronology see Morgan and Schofield (1978).

\section{Hadstock series}

Charcoal and bone from church, Hadstock, Essex $\left(52^{\circ} 4^{\prime} 43^{\prime \prime} \mathrm{N}\right.$, $0^{\circ} 16^{\prime} 30^{\prime \prime} \mathrm{E}$, Natl Grid Ref TL 55884474). All coll and subm by W J Rodwell, Old Vicarage, Chilcompton, Somerset. See Rodwell (1976).

HAR-2559. HAD2

$880 \pm 70$

Charcoal, AML 780208, id by C A Keepax as all oak from large timbers, from filling of bell-casting pit inside church. Coll Aug 1974 and subm Feb 1978.

\section{HAR-2594. HAD1}

$1080 \pm 80$

Bone, AML 780207, from colln of various animal bone fragments in Saxon domestic horizon in buried soil below church. Coll Sept 1974 and subm May 1978.

\section{HAR-2595. HAD4}

$860 \pm 70$

Human bone, AML 780210, from colln in reliquary in floor of S porticus of church. Comments: date checked by replicate measurement, HAR2697 below. (WJR): stratigraphically earlier than HAD3, below. Burial 
would seem to be Norman rather than Saxon deposit contemporary with Saxon church.

\section{HAR-2606. HAD3}

$$
\delta^{13} C=\begin{array}{r}
\mathbf{6 0 0} \pm \mathbf{8 0} \\
-19.7 \% 0
\end{array}
$$

Bone, AML 780209, from burial of child in S porticus of church. Coll Aug 1974 and subm May 1978. Comment (WJR): burial sealed filling of reliquary which contains HAD4 (HAR-2697).

\section{HAR-2697. HAD4}

$780 \pm 70$

$\delta^{13} C=-19.2 \%$

Human bone, AML 780210. Comment: replicate check measurement on HAR-2595, above.

\section{Stafford King's Pool series}

Peat from the King's Pool, Stafford, Staffordshire $\left(52^{\circ} 48^{\prime} 28^{\prime \prime} \mathrm{N}\right.$, $2^{\circ} 6^{\prime} 41^{\prime \prime} \mathrm{W}$, Natl Grid Ref SJ 925234). Coll May 1977 by J R A Greig and S M Colledge and subm Feb 1978 by S M Colledge, Dept Plant Biol, Univ Birmingham.

HAR-2577. KP100

$960 \pm 80$

AML 7716223, from $1 \mathrm{~m}$ depth within deposit. Subm Oct 1977.

HAR-2578. KP140

$920 \pm 60$

AML 7716224, from 1.4m depth within deposit. Subm Oct 1977.

HAR-2582. KP230

$1620 \pm 60$

AML 7716225, from 2.3m within deposit.

$\delta^{13} C=-28.8 \%$

General Comment (SMC): results required to date different levels of pollen diagram. Peat is most recent deposit in deep depression formed by glacial action (Colledge, 1978).

\section{North Ferriby series}

Wood, from sites assoc with Bronze Age boat finds at North Ferriby, Humberside (53 $3^{\circ} 42^{\prime} 50^{\prime \prime} \mathrm{N}, 0^{\circ} 29^{\prime} 52^{\prime \prime} \mathrm{W}$, Natl Grid Ref SE 99132524). All samples subm by Natl Maritime Mus, Greenwich, London.

\section{HAR-2759. ARC3083}

$$
\begin{aligned}
& 3150 \pm 80 \\
& \delta^{13} \mathrm{C}=-29.2 \%
\end{aligned}
$$

Oak (Quercus sp) from intertidal zone of beach. Coll May 1978 and subm July 1978 by S McGrail. Comment (SMcG): excavation will continue to investigate relationship between possible linear wooden features and site of boat finds. Sample came from same layer as Bronze Age boat dated to ca 1500 BC (Wright, 1976). 
HAR-3682. ASA3215

$$
\begin{array}{r}
\mathbf{3 5 0 0} \pm \mathbf{7 0} \\
\delta^{13} C=-27.4 \% 0
\end{array}
$$

Vertical stake, oak (Quercus sp), one of pair located either side of horizontal timber of possible trackway, hardstanding or fish weir, see McGrail (1983). Coll Aug 1979 by G T Denford and subm 1980 by G T Denford. Comment (GTD): sample from same layer as three Bronze Age boats dated to mid-2nd millennium BC, also HAR-4204, below.

\section{HAR-4204. ARCS3086}

$3420 \pm 90$

Vertical stake, hazel, from possible trackway on foreshore, (site coordinates 3355/2199). Coll and subm Dec 1980 by V E Heal. Comment (SMcG): sample $35 \mathrm{~mm}$ in diam, some bark present. Top of timber found $0.23 \mathrm{~m}$ and bottom $0.77 \mathrm{~m}$ below ground surface, penetrates $0.30 \mathrm{~m}$ into context 8 , upper $0.24 \mathrm{~m}$ in context 5 of site.

\section{HAR-2835. ARCS2213}

$$
\delta^{13} C=\begin{array}{r}
960 \pm 70 \\
-26.1 \% 0
\end{array}
$$

From remains of $\log$ boat (dugout canoe with fitted ribs) in Yorkshire Mus, York, found 1838 at Stanley Ferry, Yorkshire $\left(53^{\circ} 42^{\prime} 9^{\prime \prime} \mathrm{N}, 1^{\circ} 27^{\prime}\right.$ 38" W, Natl Grid Ref SE 35612305). Coll Aug 1978 and subm Sept 1978 by S McGrail. Comment (SMcG): date substantiates other evidence that some $\log$ boat finds are Medieval rather than prehistoric (McGrail, 1978).

\section{Somerset Levels series}

Following 33 dates are for samples coll during excavations 1975-1984 at Somerset Levels site, Somerset. Following general dates, main series is divided into sub-series (Tinney's, Baker Platform, Eclipse site, Meare Village East and Honeygore). For introduction to project and other results, see Harwell II (Otlet, 1977, p 415-416), Harwell III (Otlet \& Walker, 1979, p 360-364) and Harwell IV (Walker \& Otlet, 1985, p 77-80). Except where noted all samples coll and subm by J M Coles, Dept Archaeol, Cambridge.

\section{HAR-3078. SLP7901}

$$
\begin{array}{r}
\mathbf{4 5 9 0} \pm \mathbf{7 0} \\
\delta^{13} \mathrm{C}=-28.0 \%
\end{array}
$$

Wood, from Jones' Track, Ashcott $\left(51^{\circ} 8^{\prime} 37^{\prime \prime} \mathrm{N}, 2^{\circ} 46^{\prime} 45^{\prime \prime} \mathrm{W}\right.$, Natl Grid Ref ST 455386). Coll Feb 1979 by C R Sturdy and subm Feb 1979. Comment (JMC): one of several Neolithic structures discovered on Walton Heath (Orme, Caseldine \& Morgan, 1982), see HAR-3386, below.

\section{HAR-3195. SLP7902}

$$
\begin{array}{r}
\mathbf{5 1 8 0} \pm \mathbf{7 0} \\
\delta^{13} C=-26.8 \%
\end{array}
$$

Wood, bog oak, from Site 4, Meare Heath Field $\left(51^{\circ} 9^{\prime} 54^{\prime \prime} \mathrm{N}\right.$, $2^{\circ} 47^{\prime} 33^{\prime \prime} \mathrm{W}$, Natl Grid Ref ST 446410). Coll by R Morgan and subm March 1979. Comment (JMC): date was completely unknown but is very satisfactory as it is roughly contemporary with floating Neolithic tree-ring chronology. For description of site see Coles and Orme (1978). 
HAR-3386. SLP7904

$$
\begin{array}{r}
4420 \pm 80 \\
\delta^{13} C=-30.9 \%
\end{array}
$$

Wood, assoc with HAR-3078, above, at JN79.1, Jones' Track $\left(51^{\circ} 8^{\prime} 40^{\prime \prime} \mathrm{N}, 2^{\circ} 46^{\prime} 45^{\prime \prime} \mathrm{W}\right.$, Natl Grid Ref ST 455387). Coll and subm June 1979. Comment (JMC): same structure as HAR-3078. For description of site see Orme, Caseldine and Morgan (1982).

HAR-3387. SLP7905

$$
\begin{array}{r}
\mathbf{4 3 4 0} \pm \mathbf{8 0} \\
\delta^{13} C=-28.8 \% 0
\end{array}
$$

Wood from near Garvin's Track, Ashcott $\left(51^{\circ} 8^{\prime} 37^{\prime \prime} \mathrm{N}, 2^{\circ} 46^{\prime} 50^{\prime \prime} \mathrm{W}\right.$, Natl Grid Ref ST 454386). Coll June 1979 by B J Orme and subm June 1979. Comment (JMC): closely related to Garvin's Track (HAR-1222, -1219; see Otlet, 1979, p 361-362; Coles \& Orme, 1977; Orme, Caseldine \& Morgan, 1982).

\section{HAR-4477. SLP8103}

$$
\begin{array}{r}
\mathbf{2 8 1 0} \pm \mathbf{9 0} \\
\delta^{13} C=-25.0^{\circ} \%
\end{array}
$$

Wood from structure at complex Bronze Age site in raised bog peat at Stileway, Meare ( $51^{\circ} 9^{\prime} 52^{\prime \prime} \mathrm{N}, 2^{\circ} 45^{\prime} 55^{\prime \prime} \mathrm{W}$, Natl Grid Ref ST 465409). Coll June 1981 by K R Campbell and subm June 1981. See Orme et al (1985a) for description of site.

HAR-4739. SLP8108

$$
\begin{array}{r}
\mathbf{4 5 8 0} \pm \mathbf{7 0} \\
\delta^{13} C=-29.1 \% 0
\end{array}
$$

Wood, from structure in peat at Site 81.42, Signal Pole Ground, Ashcott Heath (51 ${ }^{\circ} 9^{\prime} 53^{\prime \prime} \mathrm{N}, 2^{\circ} 49^{\prime} 21^{\prime \prime} \mathrm{W}$, Natl Grid Ref ST 425410). Coll Nov 1981 and subm Dec 1981. See Orme et al (1985b).

\section{HAR-4998. SLP8204}

$$
\begin{array}{r}
\mathbf{3 0 2 0} \pm \mathbf{6 0} \\
\delta^{13} C=-29.8 \%
\end{array}
$$

Wood, from structure, found in bank of R Cary at Henley Bridge, Sedgemoor ( $51^{\circ} 5^{\prime} 19^{\prime \prime} \mathrm{N}, 2^{\circ} 48^{\prime} 9^{\prime \prime} \mathrm{W}$, Natl Grid Ref ST 438325). Coll and subm April 1982. Comment (JMC): date appears to confirm presumed prehistoric date (Coles \& Orme, 1983).

HAR-5054. SLP8210

$$
\begin{array}{r}
\mathbf{3 8 1 0} \pm \mathbf{7 0} \\
\delta^{13} C=-28.6 \% 0
\end{array}
$$

Peat from around flint core near line of 2nd millennium BC tracks at Ten Acres, Meare Heath $\left(51^{\circ} 9^{\prime} 50^{\prime \prime} \mathrm{N}, 2^{\circ} 47^{\prime} 44^{\prime \prime} \mathrm{W}\right.$, Natl Grid Ref ST 44374088). Coll and subm June 1982.

HAR-5086. SLP8206

$$
\begin{array}{r}
3060 \pm 70 \\
\delta^{13} C=-29.7 \% 0
\end{array}
$$

Wood, from newly discovered trackway at Godwin's Track, Sharpham $\left(51^{\circ} 8^{\prime} 31^{\prime \prime} \mathrm{N}, 2^{\circ} 45^{\prime} 48^{\prime \prime} \mathrm{W}\right.$, Natl Grid Ref ST 466384). Coll and subm July 1982. Comment (JMC): dates wooden trackway positioned near later Bronze Age complex of Tinney's Ground (Coles et al, 1985a). 
HAR-5710. SLP8301

$$
\begin{array}{r}
\mathbf{3 2 1 0} \pm \mathbf{8 0} \\
\delta^{13} C=-27.8 \% \text { o }
\end{array}
$$

Wood, from dumped brushwood on line of prehistoric route at Stileway, Meare (51 $9^{\prime} 47^{\prime \prime} \mathrm{N}, 2^{\circ} 45^{\prime} 56^{\prime \prime} \mathrm{W}$, Natl Grid Ref ST 46474076). Coll and subm July 1983 by B J Orme. Comment (JMC): relates to HAR-1221, (Otlet \& Walker, 1979, p 362) but HAR-4477 is more recent (Orme et al, 1985a).

\section{HAR-5726. SLP8307}

$$
\begin{array}{r}
\mathbf{4 6 8 0} \pm \mathbf{7 0} \\
\delta^{13} C=-30.4 \% 0
\end{array}
$$

Wood, from newly discovered hurdle in area of intense Neolithic activity at Walton Heath $\left(51^{\circ} 9^{\prime} 1^{\prime \prime} \mathrm{N}, 2^{\circ} 46^{\prime} 44^{\prime \prime} \mathrm{W}\right.$, Natl Grid Ref ST 45533935). Coll and subm Sept 1983. Comment (JMC): one of earliest hurdle-like structures in Somerset Levels. Lower in peat strata than previously known Neolithic hurdles in this area; see Orme et al (1985b).

\section{HAR-6264. SLP8401}

$$
\begin{array}{r}
\mathbf{4 6 6 0} \pm \mathbf{8 0} \\
\delta^{13} C=-29.7 \% 0
\end{array}
$$

Birch (Betula sp) from wooden structure in low peats at Foster's Walton, Walton Heath ( $51^{\circ} 8^{\prime} 56^{\prime \prime} \mathrm{N}, 2^{\circ} 46^{\prime} 45^{\prime \prime} \mathrm{W}$, Natl Grid Ref ST 455392). Coll Aug 1984 by S D Loxton and subm Aug 1984. Comment (JMC): structure, probably at least $20 \mathrm{~m}$ long, extended over two peat heads, partly destroyed by peat cutting. It formed part of traditional passageway across moors in 3rd millennium BC, with structures such as Jones', Bisgrove and Garvin's trackways serving as links in network (Orme et al, 1985b).

\section{HAR-6265. SLP8403}

$$
4790 \pm 80
$$

Hazel from rods of Neolithic hurdle at Frank's Hurdle Track, Meare Heath $\left(51^{\circ} 9^{\prime} 51^{\prime \prime} \mathrm{N}, 8^{\circ} 47^{\prime} 48^{\prime \prime} \mathrm{W}\right.$, Natl Grid Ref ST 443409). Coll Sept 1984 by S D Loxton and subm Sept 1984. Comment (JMC): hurdle is possibly oldest known in Britain.

\section{Tinney's series}

Wood from Tinney's Ground, Somerset ( $51^{\circ} 8^{\prime} 24^{\prime \prime} \mathrm{N}, 2^{\circ} 45^{\prime} 33^{\prime \prime} \mathrm{W}$, Natl Grid Ref ST 469382). For other dates in sub-series see Walker and Otlet (1985, p 79) and Coles and Orme (1980) for description of site.

\section{HAR-2773. SLP7808}

$$
\begin{array}{r}
\mathbf{2 9 6 0} \pm \mathbf{7 0} \\
\delta^{13} C=-29.7 \% 00
\end{array}
$$

From brushwood trackway intersection with oak planking and wooden artifacts. Coll July 1978 by B J Orme and subm July 1978. Comment (JMC): agrees well with dates for group of Bronze Age structures in same area.

HAR-3388. SLP7903

$$
\begin{array}{r}
\mathbf{3 8 0 0} \pm \mathbf{8 0} \\
\delta^{13} C=-28.7 \% 0
\end{array}
$$

From site 79.7 F2 $64.8 \mathrm{~m}$. Coll April 1978 by C Sturdy and subm June 
1979. Comment (JMC): indication of structures earlier than main bulk of trackways in same field, now totally destroyed.

\section{Baker Platform series}

Wood from Baker Platform, structure of Neolithic character at Westhay, Somerset $\left(51^{\circ} 10^{\prime} 36^{\prime \prime} \mathrm{N}, 2^{\circ} 49^{\prime} 4^{\prime \prime} \mathrm{W}\right.$, Natl Grid Ref ST 42854230). See Coles, Fleming and Orme (1980).

HAR-2843. SLP7810

From lowest platform. Coll and subm Sept 1975.

\section{HAR-2844. SLP7811}

From edge of platform. Coll and subm Sept 1978.
$4720 \pm 80$

$$
\delta^{13} C=-28.5 \% 0
$$

$4520 \pm 90$

HAR-2845. SLP7812

From platform. Coll and subm Sept 1978. Comment (JMC): rather early; most of Baker platform dates are late 3rd millennium BC.

HAR-2846. SLP7813

$$
\begin{array}{r}
\mathbf{4 4 5 0} \pm \mathbf{1 0 0} \\
\delta^{13} C=-28.4 \% 0
\end{array}
$$

From upper brushwood of platform. Coll and subm Sept 1978.

\section{HAR-2919. SLP7814}

$4540 \pm 80$

$$
\delta^{13} C=-30.3 \%
$$

From lower timbers of Baker platform track extension, discovered Nov 1978. Coll by S C Beckett and subm Nov 1978. Comment (JMC): dates basal structure of Neolithic track at junction with platform.

\section{HAR-2920. SLP7815}

$$
4520 \pm 70
$$

From platform track extension, discovered Nov 1978. Coll Nov 1978 by B J Orme and subm Nov 1978, relates to HAR-2919.

General Comment (JMC): all dates except HAR-2845 agree with stratigraphy of site and with BM-385 (4450 \pm 110 BP), Lu-328 (4280 \pm 65 BP) and Q$987(4230 \pm 60 \mathrm{BP})$ which date uppermost structures.

\section{Eclipse site series}

Peat samples taken to date local pollen assemblage zone boundaries E4/E5, E3/E4, and E2/E3 at Eclipse Site, Somerset $\left(51^{\circ} 9^{\prime} 41^{\prime \prime} \mathrm{N}\right.$, $2^{\circ} 47^{\prime} 17^{\prime \prime}$ W, Natl Grid Ref ST 449406). Coll Sept 1980 and subm by A E Caseldine. For description of zones see Beckett and Hibbert (1979) and Orme (1982).

HAR-4542. SLP8107

$$
\begin{array}{r}
3990 \pm 100 \\
\delta^{13} C=-26.7 \% 0
\end{array}
$$

Sphagnum/Calluna peat from $0.42 \mathrm{~m}$ below present ground surface. 
Subm July 1981. Comment (JMC): provides essential date for pollen zone boundary E4/E5 and marks beginning of increased clearance activity in area. Eclipse track was constructed during this phase.

HAR-4543. SLP8105

$$
\begin{aligned}
& 4230+80 \\
& \delta^{13} \mathrm{C}=-28.1 \%
\end{aligned}
$$

Sphagnum/Calluna/Eriophorum peat from 0.70 to $0.71 \mathrm{~m}$ below present ground surface. Subm Aug 1981. Comment (JMC): dates E3/E4 boundary, regional assemblage zone boundary $\mathrm{C} / \mathrm{D}$, end of forest regeneration phase.

HAR-4544. SLP8106

$$
\begin{array}{r}
4640 \pm 70 \\
\delta^{13} C=-27.6 \% 0
\end{array}
$$

Calluna/Eriophorum peat from 0.94 to $0.95 \mathrm{~m}$ below present ground surface. Subm Aug 1981. Comment (JMC): dates E2/E3 boundary, regional assemblage zone boundary $\mathrm{B} / \mathrm{C}$, end of 1 st major clearance in area.

HAR-4865. SLP8109

$5440 \pm 70$

$$
\delta^{13} C=-27.4 \%
$$

From base of peat monolith at E80.212 (Natl Grid Ref ST 449406). Subm Feb 1982. Comment (JMC): dates clay-peat interface.

General Comment (JMC): dates are earlier than those obtained for probably comparable boundaries at Meare Heath, Abbot's Way and Sweet Track factory site.

\section{Meare Village East series}

Charcoal from Meare Village East, Somerset $\left(51^{\circ} 10^{\prime} 33^{\prime \prime} \mathrm{N}\right.$, $2^{\circ} 47^{\prime} 28^{\prime \prime} \mathrm{W}$, Natl Grid Ref ST 447422). Coll April 1982 by B J Orme and subm April 1982.

HAR-5000. SLP8203

Context 82.1119.

HAR-5001. SLP8201

Context 82.1073.

HAR-5002. SLP8202

Context 82.1094.

$$
\begin{array}{r}
\mathbf{2 0 8 0} \pm \mathbf{6 0} \\
\delta^{13} C=-26.9 \% 0
\end{array}
$$

$1740 \pm 60$

$\delta^{13} C=-27.4 \%$ o

$$
\begin{array}{r}
\mathbf{2 0 9 0} \pm \mathbf{7 0} \\
\delta^{13} C=-27.0 \%
\end{array}
$$

General Comment (JMC): samples used to date stratigraphic context in Iron Age site. HAR-5001 is from same broad context as HAR-5000; -5002 is from lower layer in same sequence, $i e$, all three samples are from same mound. For description of site see Orme, Coles and Silvester (1983).

\section{Honeygore series}

Samples from tracks excavated in Neolithic peat at Honeygore 
Complex, Somerset Levels $\left(51^{\circ} 10^{\prime} 51^{\prime \prime} \mathrm{N}, 2^{\circ} 50^{\prime} 8^{\prime \prime} \mathrm{W}\right.$, Natl Grid Ref ST 416428). For description of site see Coles et al (1985b).

HAR-5721. SLP8302

$$
\begin{array}{r}
\mathbf{4 6 1 0} \pm \mathbf{9 0} \\
\delta^{13} C=-28.2 \% 0
\end{array}
$$

Wood, from Honeygore track. Coll Aug 1983 by A E Caseldine and subm Aug 1983. Comment (JMC): slightly younger than previous dates which centered on ca 2800 BC (Coles et al, 1985b).

HAR-5722. SLP8303

$4610 \pm 100$

Wood from hurdle A of Honeybee track. Coll and subm Aug 1983. Comment (JMC): $c f$ HAR-5723.

HAR-5723. SLP8304

$4500 \pm 70$

$$
\delta^{13} \mathrm{C}=-28.3 \%
$$

Wood from hurdle B of Honeybee track. Coll and subm Aug 1983. Comment (JMC): see HAR-5722, above, with which these is good correlation.

HAR-5724. SLP8305

$$
\begin{array}{r}
\mathbf{4 5 6 0} \pm \mathbf{8 0} \\
\delta^{13} C=-28.6^{0} \%
\end{array}
$$

Wood from complex of Honeycat track. Coll and subm Aug 1983. Comment (JMC): slightly older than previous dates: HAR-653, -652 (Otlet, 1977, p 415) and Q-427, -429, -320; but see discussion in Coles et al (1985b).

\section{HAR-5727. SLP8306}

$$
\begin{array}{r}
\mathbf{4 3 0 0} \pm \mathbf{7 0} \\
\delta^{13} C=-27.5 \% 0
\end{array}
$$

Peat, from thin peat surface underlying hurdle B of Honeybee track. Coll Aug 1983 and subm Sept 1983. Comment (JMC): younger than wood overlying HAR-5723.

\section{Priory Barn series}

Wood from Augustinian Priory excavation, Taunton, Somerset $\left(51^{\circ} 1^{\prime} 4^{\prime \prime} \mathrm{N}, 3^{\circ} 5^{\prime} 57^{\prime \prime} \mathrm{W}\right.$, Natl Grid Ref ST 229249$)$. Coll 1978 by P Leach and subm July 1978 by J Hillam, Dept Prehistory and Archaeol, Univ Sheffield.

HAR-2804. TAUN40A

$$
\delta^{13} C=\begin{array}{r}
\mathbf{8 4 0} \pm \mathbf{9 0} \\
-26.4 \%
\end{array}
$$
Layer 40.

AML 781642, 7-year-old ash twig, part of bulk wood sample from

HAR-2806. TAUN96

$$
\begin{array}{r}
1090 \pm \mathbf{8 0} \\
\delta^{13} C=-28.3 \% 0
\end{array}
$$

AML 781641, oak from Layer 96. Comment (JH): growth allowance not known but contained at least 20 growth rings. 
HAR-2815. TAUN40B

AML 781643, willow/poplar twig or branch ca 17 years old, part of bulk wood sample from Layer 40 .

\section{Ledston series}

Samples from Ledston, West Yorkshire $\left(53^{\circ} 45^{\prime} 35^{\prime \prime} \mathrm{N}, 1^{\circ} 20^{\prime} 35^{\prime \prime} \mathrm{W}\right.$, Natl Grid Ref SE 433295). Coll Sept 1976 by J Keighley and I Hodder, subm Aug 1978 by R E Yarwood, West Yorkshire Co Council, Wakefield.

HAR-2805. LEDSF363

$2080 \pm 100$

Bone, AML 781940, from burial sealed in bottom of storage pit (F704).

$$
\delta^{13} \mathrm{C}=-22.0 \%
$$

$2270 \pm 70$

HAR-2825. LEDSF418

$$
\delta^{13} C=-24.6 \%
$$
(F539).

Charcoal, AML 781939, from sample sealed in fill of post hole

General Comment (REY): these are $1 \mathrm{st}{ }^{14} \mathrm{C}$ dates for prehistoric site on West Yorkshire limestone. They confirm Iron Age occupation of Ledston and, together with crop mark evidence for this area, suggest extensive prehistoric settlement.

\section{Silver St series}

Oak samples, taken as part of dendrochronologic study, from Silver St, Glastonbury, Somerset $\left(51^{\circ} 8^{\prime} 48^{\prime \prime} \mathrm{N}, 2^{\circ} 42^{\prime} 48^{\prime \prime} \mathrm{W}\right.$, Natl Grid Ref ST 501389). Coll 1978 by R H Leech and subm July 1978 by J Hillam.

HAR-2812. GL263

$$
\begin{array}{r}
1340 \pm 70 \\
\delta^{13} C=-25.2 \% 0
\end{array}
$$

AML 781638, ca 20 rings from sample containing 38 rings taken close to outer edge of tree.

HAR-2813. GL239

$1450 \pm 80$

$\delta^{13} C=-26.7 \%$

AML 781639, from rings 15 to 34 of 79-year-old sample, probably from near outer edge of tree, although no sapwood present.

\section{HAR-2814. GL249B}

$1470 \pm 80$

AML 781640, from sample containing ca 20 rings. Comment (JH): not known if from inner or outer rings.

General Comment $(\mathrm{JH})$ : dendrochronology suggests that timbers 239 (HAR2813) and 263 (HAR-2812) are contemporary but too few rings present to be certain. 


\section{Barton series}

Wood id by C A Keepax as oak (Quercus sp) from St Peter's Church, Barton-on-Humber ( $53^{\circ} 41^{\prime} 1^{\prime \prime} \mathrm{N}, 0^{\circ} 25^{\prime} 59^{\prime \prime} \mathrm{W}$, Natl Grid Ref TA 03472195). Coll July 1978 and subm Aug 1978 by W J Rodwell, Old Vicarage, Chilcompton, Somerset. Site is described in Rodwell and Rodwell (1982).

\section{HAR-2863. BHO5}

$$
\delta^{13} C=\begin{array}{r}
\mathbf{7 2 0} \pm \mathbf{6 0} \\
-25.5 \% 0
\end{array}
$$

AML 781906, from stump of beam embedded in belfry wall. Comment (WJR): wall supported former 13 th to 14 th century spire, probably contemporary with HAR-2864, below.

HAR-2864. BHO6

$$
\mathbf{7 8 0} \pm \mathbf{8 0}
$$

AML 781907 from central joist in floor of ringing chamber of tower. Comment (WJR): floor probably contemporary with HAR-2863, above.

HAR-2865. BHO3

$$
\begin{array}{r}
\mathbf{5 7 0} \pm \mathbf{7 0} \\
\delta^{13} C=-25.0 \% \text { o }
\end{array}
$$

AML 781904, from E-W timber of base frame of former 13th to 14th century spire.

\section{HAR-3106. BHO1}

$$
960 \pm 70
$$$$
\delta^{13} C=-26.0 \%
$$

AML 781902, from mature timber. Comment (WJR): joist fragment from original Saxon timber supporting floor of upper chamber in AngloSaxon W annex of church.

\section{Mucking-Central Unit series}

Charcoal from recut Iron/Bronze Age ring ditch at North Ring, Mucking, Essex (51 $30^{\circ} 13^{\prime \prime} \mathrm{N}, 0^{\circ} 24^{\prime} 52^{\prime \prime} \mathrm{E}$, Natl Grid Ref TQ 67558112). Coll July 1978 by D Bond and subm Oct 1978 by N D Balaam, Ancient Monuments Lab. For description of site see Bond (1980).

HAR-2893. 23-1658

$$
\begin{array}{r}
\mathbf{2 6 3 0} \pm \mathbf{1 1 0} \\
\delta^{13} C=-24.2 \% 0
\end{array}
$$

AML 7824847, id by C A Keepax as Acer sp (mature timbers) and some Prunus sp, overlying primary fill.

HAR-291 1. 23-34

$$
\begin{array}{r}
\mathbf{2 7 0 0} \pm \mathbf{8 0} \\
\delta^{13} C=-24.6 \% 0
\end{array}
$$

AML 7820949, id by C A Keepax as Acer sp, Quercus sp and Corylus/ Alnus sp, from mature timbers, from primary fill.

\section{Warbleton series}

Charcoal from iron smelting bloomery furnace at Warbleton Parish, Turners Green, Heathfield, East Sussex $\left(50^{\circ} 57^{\prime} 5^{\prime \prime} \mathrm{N}, 0^{\circ} 20^{\prime} 11^{\prime \prime} \mathrm{E}\right.$, Natl 
Grid Ref TQ 64091954). Coll Nov 1978 by W R Beswick and O Bedwyn and subm Nov 1978 by W R Beswick, Turners House, Heathfield, Sussex. Site is described in Beswick (1978, 1979).

HAR-2930. TG2

$1810+70$

$\delta^{13} C=-26.3 \%$

AML 785542, id by C A Keepax as oak (Quercus sp) from mature timbers, from reaction zone of furnace still containing some charge.

HAR-2932. TG4

$2040 \pm 70$

$\delta^{13} C=-25.1 \%$

AML 785543, id by C A Keepax as oak (Quercus sp) from mature timbers, from same context as HAR-2930, above.

HAR-3017. TG3

$1900 \pm 70$

$\delta^{13} C=-24.8 \%$

AML 785541, from smithy area $4.8 \mathrm{~m}$ from furnace, $0.62 \mathrm{~m}$ below cultivation surface.

General Comment (WRB): HAR-2930, -2932, and -3017 are consistent with dates for two other sites with similar unusual design of bloomery furnaceLevisham, East Yorkshire and Engsbach, Siegerland, West Germany (Rutter \& Hayes, 1970; Hayes, 1983; Weierhausen, 1939). Dates confirm smithy area was contemporaneous with furnace.

\section{Condicote series}

Charcoal from early secondary fill of inner ditch of henge monument at Condicote, Gloucestershire ( $51^{\circ} 57^{\prime} 13^{\prime \prime} \mathrm{N}, 1^{\circ} 46^{\prime} 34^{\prime \prime} \mathrm{W}$, Natl Grid Ref SP 15382841). Coll 1977 and subm 1978 by A Saville, Art Gallery \& Mus, Cheltenham. For description of site see Saville (1983).

\section{HAR-3064. CH77C69A}

$$
\begin{array}{r}
\mathbf{3 7 2 0} \pm \mathbf{8 0} \\
\delta^{13} C=-28.0 \% 0
\end{array}
$$

AML 790631, id by C A Keepax as hawthorn (Rosaceae subfamily Pomoideae) and hazel or alder (Corylus sp) from mature timbers, in Layer 9A. Comment (AS): sample assoc with prehistoric pottery of Beaker-related type.

\section{HAR-3067. CH77C114}

$3670 \pm 100$ bers, in Layer 14.

\section{Great Linford series}

Wood id by C A Keepax as oak (Quercus sp) from mature timbers, from buried supports of Medieval Post Mill (Site 3709) at Great Linford, Buckinghamshire (52 $2^{\prime} 12^{\prime \prime} \mathrm{N}, 0^{\circ} 45^{\prime} 7^{\prime \prime} \mathrm{W}$, Natl Grid Ref SP 85544208). Coll Sept 1977 and subm Feb 1979 by R J Zeepvat, Bradwell Abbey Field Centre, Milton Keynes (Zeepvat, 1980). 
HAR-3121. M1098W1B

AML 790636.

HAR-3122. M1098W1A

AML 790635. $\delta^{13} C=-25.5 \% 0$

$730 \pm 80$

$\delta^{13} C=-25.6 \%$

General Comment (RJZ): beam appears to have measured $0.3 \mathrm{~m} \times 0.3 \mathrm{~m} \times$ $5.5 \mathrm{~m}$. It was packed with stone in cross-shaped trench. Samples originally from near center of beam.

\section{Rivington-Anglezarke series}

Peat taken as part of paleo-ecologic studies on Rivington and Anglezarke Moors, Lancashire. Human exploitation of area dates from Mesolithic/Neolithic (flint finds and barrow, Bu'lock, 1958) through Historic period to present. Samples coll and subm by M G Bain and B Barnes, Bolton Inst Higher Educ, Yorkshire. Dates were required to support strat and palynol evidence and provide chronol framework for record. Similar studies are described in Hibbert, Switzur and West (1971) and Tallis and McGuire (1972).

\section{HAR-6206. WH2}

$$
\begin{array}{r}
\mathbf{2 9 4 0} \pm \mathbf{7 0} \\
\delta^{13} C=-29.4 \% 0
\end{array}
$$

AML 832900, from 1.17 to $1.20 \mathrm{~m}$ depth, well-marked clearance phase with cultivation and possible relation to metal extraction, at Winter Hill pollen site, Rivington Moor ( $53^{\circ} 37^{\prime} 39^{\prime \prime} \mathrm{N}, 2^{\circ} 31^{\prime} 7^{\prime \prime} \mathrm{W}$, Natl Grid Ref SD 657147). Coll Oct 1979. Comment (BB): date corresponds well with estimates based on peat accumulation rates in other local profiles, and marks beginning of sustained clearance activity in Rivington-Anglezarke uplands.

\section{HAR-6207. BB1}

$\mathbf{5 6 6 0} \pm \mathbf{8 0}$

AML 832869, from 2.42 to $2.46 \mathrm{~m}$ depth, peat initiation and clearance phase, at Black Brook pollen site, Anglezarke Moor $\left(53^{\circ} 39^{\prime} 41^{\prime \prime} \mathrm{N}\right.$, $2^{\circ} 33^{\prime} 25^{\prime \prime} \mathrm{W}$, Natl Grid Ref SD 632185). Coll Nov 1980. Comment (BB): date agrees well with initial estimates of age (Mesolithic, ie, ca $5500 \mathrm{BP}$ ) based on palynol and strat data. Marks earliest forest clearance phase yet detected in area.

HAR-6208. PKA1

$$
\begin{array}{r}
\mathbf{3 1 0} \pm \mathbf{8 0} \\
\delta^{13} C=29.6 \% 0
\end{array}
$$

AML 832897, id as sphagnum/monocot from 0.35 to $0.37 \mathrm{~m}$ depth at Pikestones pollen site, Anglezarke Moor ( $53^{\circ} 38^{\prime} 53^{\prime \prime} \mathrm{N}, 2^{\circ} 33^{\prime} 41^{\prime \prime} \mathrm{W}$, Natl Grid Ref SD 629170). Coll Nov 1980. Comment (BB): date is younger than expected (Norse or Medieval) but accords well with documentary evidence for increased agric activity in 16 th and 17 th centuries AD on uplands. Date reinforces palynol evidence of truncated profile. 
HAR-6209. PK1

$$
\begin{array}{r}
1710 \pm 70 \\
\delta^{13} C=-29.4 \% o
\end{array}
$$

AML 832898, from 0.4 to $0.45 \mathrm{~m}$ depth at Pikestones pollen site, Anglezarke Moor (53 $38^{\prime} 59^{\prime \prime} \mathrm{N}, 2^{\circ} 33^{\prime} 52^{\prime \prime} \mathrm{W}$, Natl Grid Ref SD 627172). Coll Nov 1980. Comment (BB): dates peat initiation on flanks of moor, with clearance phenomena. Romano-British date is earlier than expected (ca $1000 \mathrm{BP}$ ) but there are problems in estimating age based on accumulation rates in profile. Result corresponds with clearances dated from Round Loaf samples (HAR-621 1, -6212, below). Palynol evidence from both sites suggests considerable upland exploitation at this time including both arable and pastoral activity.

\section{HAR-6210. BB2}

$4740 \pm 70$

AML 832899 , from 2.20 to $2.24 \mathrm{~m}$ depth, early clearance phase with evidence of elm decline, at Black Brook pollen site, Anglezarke Moor (533 $36^{\prime} 59^{\prime \prime} \mathrm{N}, 20^{\circ} 33^{\prime} 23^{\prime \prime} \mathrm{W}$, Natl Grid Ref SD 632135). Coll Nov 1980. Comment (BB): agrees well with accumulation rate estimates from Round Loaf site and marks 'Elm decline horizon' within published range for NW England.

HAR-6211. RL3

$$
\begin{array}{r}
1550 \pm 70 \\
\delta^{13} C=-28.9 \%
\end{array}
$$

AML 832901, id as Calluna eriophorum, from 0.50 to $0.55 \mathrm{~m}$ depth, horizon marking end of well-defined clearance phase at Round Loaf pollen site, Anglezarke Moor ( $53^{\circ} 39^{\prime} 25^{\prime \prime} \mathrm{N}, 2^{\circ} 33^{\prime} 14^{\prime \prime} \mathrm{W}$, Natl Grid Ref SD 634180). Coll Aug 1980. Comment (BB): although earlier than estimated (ca $900 \mathrm{BP}$ ), date relates well-marked clearance and agric episode to RomanoBritish period and accords well with RL4 (HAR-6212).

\section{HAR-6212. RL4}

$1710 \pm 70$

AML 832902, id as Calluna eriophorum, from 0.60 to $0.65 \mathrm{~m}$ depth, beginning of well-defined clearance phase, at Round Loaf pollen site,

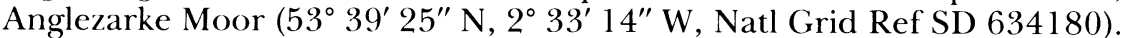
Coll Aug 1980. Comment (BB): date agrees well with RL3 (HAR-6211, above) and marks initiation of well-defined Romano-British upland clearance horizon. This corresponds with clearance evidence of similar date (see HAR-6209, above) from Pikestones pollen site.

\section{REFERENCES}

Anonymous, 1973, Wiltshire Archaeol Magazine, v 68, p 57.

Beckett, S C and Hibbert, F A, 1979, Vegetational change and the influence of prehistoric man in the Somerset Levels: New Phytologist, v 83, p 577-600.

Beswick, W R, 1978, A note on early ironmaking in Sussex: Sussex Industrial Hist, v 8, p 23. 1979, Ironmaking origins and their early impact on the English Weald: Sussex Industrial Hist, v 9 , p 10.

Bond, D, 1980, North Ring, Mucking: an enclosure of the Late Bronze Age: East Anglian Archaeol Repts (Essex). 
Bu'lock, J D, 1958, The Pikestones, a chambered long cairn of Neolithic type on Anglezarke Moors, Lancashire: Trans Lancashire Cheshire Antiquarian Soc, no. 68, p 143-145.

Carver, M O H, Donaghey, S and Sumpter, A B, 1978, The Colonia: Riverside structures and a well in Skeldergate and buildings in Bishophill: The Archaeol of York, v 4, no. 1, p 25.

Chowne, P, 1980, Bronze Age settlement in South Lincolnshire, in Barrett, J C and Bradley, R J, eds, Settlement and society in the Later Bronze Age: British Archaeol Repts, v 83.

Coles, J M, Fleming, A M and Orme, B J, 1980, The Baker site: a Neolithic platform: Somerset Levels Papers, v 6, p 6-23.

Coles, J M and Orme, B J, 1977, Garvin's tracks: Somerset Levels Papers, v 3, p 73-81. 1978, The Meare Heath track: Somerset Levels Papers, v 4, p $11-39$. 1980, Tinney's Ground 1978-1979: Somerset Levels Papers, v 6, p 61-68. 1983, The Sedgemoor survey 1982: Somerset Levels Papers, v 9, p 6-8.

Coles, J M, Orme, B J, Caseldine, A E and Morgan, R A, 1985a, Godwin's track: a Bronze Age structure at Sharpham: Somerset Levels Papers, v 11 , p 69-74. $11, \mathrm{p} 51-61$.

Colledge, S M, 1978, An interim environmental report, the King's Pool, Stafford: Ancient Monuments Lab Rept, no. 2535.

Donaghey, S, 1986, '58-9 Skeldergate', in Moulden, J and Tweddle, D, eds, Anglo-Scandinavian settlement south-west of the Ouse: The Archaeol of York, v 8, no. 1, p 37-48.

Fasham, P J, 1980, Excavations on Bridget's and Burntwood Farms, Itchen Valley Parish, Hampshire, 1974: Hants Field Club Archaeol Soc Proc, v 36, p 37-86.

Green, C J S, 1971, Interim report on excavations in the Roman cemetery, Poundbury, Dorchester, 1970: Dorset Nat Hist Archaeol Soc Proc.

- 1974, Interim report on excavation in the Roman cemetery, Poundbury, Dorchester, 1973: Dorset Nat Hist Archaeol Soc Proc, v 95, p 100.

1976, Poundbury, in Dorset archaeol in 1976: Dorset Nat Hist Archaeol Soc Proc, v 98, p 55-56.

1982, The cemetery of a Roman-British Christian community at Poundbury, Dorchester, Dorset, in Pearce, S M, ed, The early church in Western Britain and Ireland: British Archeol Research Repts, v 102.

Hall, A R, Kenward, H K and Williams, D, 1980, The past environment of York: Environmental evidence from Roman deposits in Skeldergate: The Archaeol of York, v 14, no. 3, p 105.

Hayes, R H, 1983, Levisham Moor archaeologic investigation 1957-1978: North Yorks Moors Nat Park Comm/Scarborough Archaeol Hist Soc, p 24.

Hibbert, F A, Switzur, V R and West, R G, 1971, Radiocarbon dating of Flandrian pollen zones at Red Moss, Lancashire: Royal Soc [London] Proc B, v 177, p 161-176.

MacGregor, H, in press, Structures on the north-east side of Aldwark, in Hall, R A, MacGregor, $\mathrm{H}$ and Stockwell, $\mathrm{M}$, eds, Medieval tenements in Aldwark and other sites: The Archaeol of York, v 10, no. 2.

McGrail, S, 1978, Logboats of England and Wales: Natl Maritime Mus Archaeol ser no. 2, Br Archaeol Repts, v 51, p 275-276.

- 1981, A medieval logboat from the River Calder at Stanley Ferry: Medieval Archaeol, v 25, p 160-164.

- 1983, Interpretation of archaeol evidence for maritime structures, in Annis, $\mathbf{P}$, ed, Sea studies: Natl Maritime Mus, p 33-46.

Mercer, R J, 1980, Hambledon Hill, a Neolithic landscape: Edinburgh, Edinburgh Univ Press.

1985, A Neolithic fortress and funeral gate: Scientific Am, v 252, no. 3, p 94101.

Morgan, R A and Schofield, J, 1978, Tree rings and the archaeol of the Thames waterfront in the City of London, in Fletcher, J M, ed, Dendrochronology in Europe: Council $\mathrm{Br}$ Archaeol Repts, Internatl ser, v 51, p 223-238.

Orme, B J, 1982, The use of radiocarbon dates from the Somerset Levels: Somerset Levels Papers, v 8, p $9-25$.

Orme, B J, Caseldine, A E and Morgan, R A, 1982, Recent discoveries on Walton Heath: Garvin's, Bisgrove's and Jones' tracks: Somerset Levels Papers, v 8, p 51-64.

Orme, B J, Coles, J M and Silvester, R J, 1983, Meare Village East 1982: Somerset Levels Papers, v 9, p 49-74.

Orme, B J, Coles, J M, Caseldine, A E and Morgan, R A, 1985a, A later Bronze Age complex at Stileway: Somerset Levels Papers, v 11, p 75-79. v 11, p $62-68$

Otlet, R L, 1977, Harwell radiocarbon measurements II: Radiocarbon, v 19, no. 3, p 400423 . 
Otlet, R L, 1979, An assessment of errors in liquid scintillation methods ${ }^{2}{ }^{14} \mathrm{C}$ dating, in Berger, $\mathrm{R}$ and Suess, $\mathrm{H}$ E, eds, Radiocarbon dating, Internatl ${ }^{14} \mathrm{C}$ conf, 9 th, Proc: Berkeley, Univ California Press, p 256-267.

Otlet, R L and Walker, A J, 1979, Harwell radiocarbon measurements III: Radiocarbon, v 21, no. 3, p 358-383.

1983, The computer writing of radiocarbon reports and further developments in the storage and retrieval of archaeological data, in Mook, W G and Waterbolk, H T, eds, ${ }^{14} \mathrm{C}$ and archaeology, Internatl symposium, 1 st, Proc: PACT, v 8, p 91-106.

Otlet, R L and Warchal, R M, 1978, Liquid scintillation counting of low-level ${ }^{14} \mathrm{C}$, in Crook, $\mathrm{M} \mathrm{A}$ and Johnson, P, eds, Liquid scintillation counting, vol 5: London, Heyden, p 210 218.

Rodwell, W J, 1976, The archaeological investigation of Hadstock Church Essex: Antiquaries Jour, v 56, p 55-71.

Rodwell, W J and Rodwell, K A, 1982, St Peter's Church, Barton-upon-Humber: Excavation and structural study 1978-1981: Antiquaries Jour, v 62, p 283-315.

985 , Rivenhall: investigations on the Roman villa, church and village: Council Br Archaeol Res Rpts, Br ser, v 55.

Rutter, J G and Hayes, R H, 1970, A pre-Roman iron-smelting site at Levisham, North Yorks: Bull Hist Metallurgy Soc, v 4, no. 2, p 79.

Saville, A, 1983, Excavations at Condicote Henge monument, Gloucestershire 1977: Trans Bristol Gloucester Archaeol Soc, v 101, p $21-47$.

Smith, K, 1979, The excavation of Winklebury Camp, Basingstoke Hants: Prehist Soc Proc, v 43.

Stead, I M, 1977, La Tène burials between Burton Fleming and Rudston, North Humberside: Antiquaries Jour, v 56, pt II, p 224-225.

Tallis, J H and McGuire, J, 1972, Central Rossendale: the evolution of an upland vegetation. I. The clearance of Woodland: Jour Ecology, v 60, p 721-737.

Tatton-Brown, T, 1974, Excavations at the Custom House site, City of London 1973: Trans London Middlesex Archaeol Soc, v 25, p 117-219.

Walker, A J and Otlet, R L, 1985, Harwell radiocarbon measurements IV: Radiocarbon, v 27, no. $1, \mathrm{p} 74-94$

Weierhausen, P, 1939, Vorgeschichtliche eisenhutten im Deutschlands: Leipzig, p 9.

Wright, E V, 1976, The North Ferriby boats: Natl Maritime Mus, Mon no. 23.

Zeepvat, R J, 1980, Post mills in archaeology: Current Archaeol, v 71, p 375-377. 